\title{
The Charge Conjugation Quantum Number in Multiquark Systems
}

\author{
Fl. Stancu \\ Université de Liège, Institut de Physique B5, Sart Tilman, B-4000 Liège 1, Belgium \\ E-mail: fstancu@ulg.ac.be ${ }^{1}$
}

\begin{abstract}
.
We discuss the charge conjugation quantum number for tetraquarks and meson-meson molecules, both seen as possible interpretations of the newly found $X Y Z$ charmonium-like resonances.
\end{abstract}

Keywords: Charge Conjugation, Exotic Resonances

PACS: $11.30 . \mathrm{Er}, 14.20 . \mathrm{Kp}$

\section{INTRODUCTION}

The discovery by Belle [1] of the very narrow narrow $X(3872)$ resonance has revived the interest in heavy quarkonium, both experimentally and theoretically. The observation of $X(3872)$ has been confirmed by CDF [2], D0 [3] and Babar Collaborations [4]. Other charmonium-like states, as for example $X(3940)$ [5], $Y(4260)$ [6] and $Z^{+}$(4430) [7] have been uncovered in B-factory experiments. A partial list is shown in Table 1. Several review papers, as for example [8, 9], discuss the difficulty of interpreting these resonances as charmonium states. Seen as exotics, they can possibly be tetraquarks, meson-meson molecules, hybrids, glueballs, etc.

TABLE 1. Properties of newly discovered charmonium-like resonances

\begin{tabular}{|c|c|c|c|c|}
\hline Resonance & $\begin{array}{c}\text { Mass } \\
(\mathrm{MeV})\end{array}$ & $\begin{array}{c}\text { Width } \\
(\mathrm{MeV})\end{array}$ & $\mathrm{J}^{P C}$ & Decay modes \\
\hline $\mathrm{X}(3872)$ & $3871.4 \pm 0.6$ & $<2.3$ & $1^{++}$ & $\pi^{+} \pi^{-} J / \Psi, \gamma J / \Psi$ \\
$\mathrm{X}(3940)$ & $3942 \pm 9$ & $37 \pm 17$ & $\mathrm{~J}^{P+}$ & $D \bar{D}^{*}$ \\
$\mathrm{Y}(3940)$ & $3943 \pm 17$ & $87 \pm 34$ & $\mathrm{~J}^{P+}$ & $\omega J / \Psi$ \\
$\mathrm{Z}(3930)$ & $3929 \pm 5$ & $29 \pm 10$ & $2^{++}$ & $D \bar{D}$ \\
$\mathrm{X}(4160)$ & $4156 \pm 29$ & $139_{-65}^{+113}$ & $\mathrm{~J}^{P+}$ & $D^{*} \bar{D}^{*}$ \\
$\mathrm{Y}(4260)$ & $4264 \pm 12$ & $83 \pm 22$ & $1^{--}$ & $\pi^{+} \pi^{-} J / \Psi$ \\
$\mathrm{Z}^{+}(4430)$ & $4433 \pm 5$ & $45_{-18}^{+35}$ & $?$ & $\pi^{ \pm} \Psi^{\prime}$ \\
$\mathrm{Y}(4660)$ & $4664 \pm 12$ & $48 \pm 15$ & $1^{--}$ & $\pi^{+} \pi^{-} \Psi^{\prime}$ \\
\hline
\end{tabular}

${ }^{1}$ Based on a talk given at the Joint Meeting Heidelberg-Liège- Paris-Wroclaw (HLPW08): Three Days of Strong Interactions and Astrophysics, Spa, Liège, March 6-8, 2008 
Whatever picture is adopted, it is important to properly determine the charge conjugation number $C$ of the resonance. From Table 1 one can see that $C$ is known in nearly all cases. Here we shall discuss the tetraquark option, for example [10], and the molecular option, for example [11] or [12], where we noticed that some difficulty has been encountered in defining it.

\section{THE BASIS STATES}

We recall the definition of tetraquark basis states as given in Refs. [13, 14, 15]. This basis can provide a direct connection with the diquark-antidiquark states [16] and also with the molecular states [11, 12, 17].

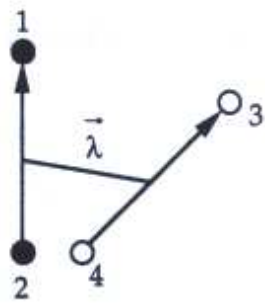

(a)

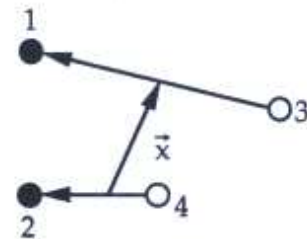

(b)

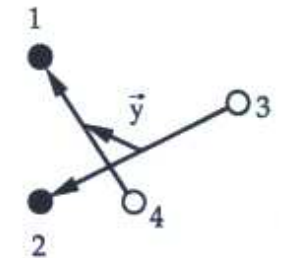

(c)

FIGURE 1. Three independent relative coordinate systems. Solid and open circles represent quarks and antiquarks respectively: (a) diquark-antidiquark channel, (b) direct meson-meson channel, (c) exchange meson-meson channel.

In Fig. 1 we suppose that the particle 1 is the charmed quark $c$, the particle 2 a light quark $q=u, d$, particle 3 the anticharmed quark $\bar{c}$ and particle 4 a light antiquark $\bar{q}=\bar{u}, \bar{d}$.

The total wave function of a tetraquark is a linear combination of products of orbital, spin, flavour and colour parts. We shall successively introduce the orbital, colour and spin parts of the wave function. The flavor part is fixed.

\subsection{The orbital part}

There are at least three possible ways to define the relative coordinates. The three relevant possibilities for our problem are shown in Fig. 1. In the cases $(a),(b)$ and $(c)$ the internal coordinates are

$$
\begin{array}{lll}
\vec{\sigma}=\frac{1}{\sqrt{2}}\left(\overrightarrow{r_{1}}-\overrightarrow{r_{2}}\right), & \overrightarrow{\sigma^{\prime}}=\frac{1}{\sqrt{2}}\left(\overrightarrow{r_{3}}-\overrightarrow{r_{4}}\right), \quad \vec{\lambda}=\frac{1}{2}\left(\overrightarrow{r_{1}}+\overrightarrow{r_{2}}-\overrightarrow{r_{3}}-\overrightarrow{r_{4}}\right), \\
\vec{\rho}=\frac{1}{\sqrt{2}}\left(\overrightarrow{r_{1}}-\overrightarrow{r_{3}}\right), \quad \overrightarrow{\rho^{\prime}}=\frac{1}{\sqrt{2}}\left(\overrightarrow{r_{2}}-\overrightarrow{r_{4}}\right), & \vec{x}=\frac{1}{2}\left(\overrightarrow{r_{1}}-\overrightarrow{r_{2}}+\overrightarrow{r_{3}}-\overrightarrow{r_{4}}\right), \\
\vec{\alpha}=\frac{1}{\sqrt{2}}\left(\overrightarrow{r_{1}}-\overrightarrow{r_{4}}\right), \quad \overrightarrow{\alpha^{\prime}}=\frac{1}{\sqrt{2}}\left(\overrightarrow{r_{2}}-\overrightarrow{r_{3}}\right), & \vec{x}=\frac{1}{2}\left(\overrightarrow{r_{1}}-\overrightarrow{r_{2}}-\overrightarrow{r_{3}}+\overrightarrow{r_{4}}\right) .
\end{array}
$$


The first system of coordinates is convenient when the quarks or antiquarks are correlated to form diquarks, as in the diquark-antidiquark model. The coordinates (2) and (3) called direct and exchange meson-meson channels, are useful in describing strong decays or introduce a molecular picture [17], provided a quark structure is imposed at short separations. One should use the system which is more appropriate for a given problem. But in specific calculations one can pass from one coordinate system to the other by orthogonal transformations [14].

\subsection{The colour part}

In the colour space one can construct a colour singlet tetraquark state using intermediate couplings associated to the three coordinate systems defined above. In this way one obtains three distinct bases [15]

$$
\begin{array}{ll}
\left|\overline{3}_{12} 3_{34}\right\rangle, & \left|6_{12} \overline{6}_{34}\right\rangle, \\
\left|1_{13} 1_{24}\right\rangle, & \left|8_{13} 8_{24}\right\rangle, \\
\left|1_{14} 1_{23}\right\rangle, & \left|8_{14} 8_{23}\right\rangle .
\end{array}
$$

In the basis (4) the states 3 and $\overline{3}$ are antisymmetric and 6 and $\overline{6}$ are symmetric under interchange of quarks or antiquarks. This basis is convenient in a diquarkantidiquark picture. In Ref. [16] only the $\left|\overline{3}_{12} 3_{34}\right\rangle$ state has been considered which restricts the spectrum to half of the allowed states [18]. The sets (5) or (6) $)$, contain a singlet-singlet colour and an octet-octet colour state. The amplitude of the latter vanishes asymptotically when a tetraquark decays into two mesons. These are called hidden colour states, by analogy to states which appear in the nucleon-nucleon problem. When the amplitude of a hidden colour state is far the most dominant in the wave function, the corresponding open channel acquires, in exchange, a tiny value for its amplitude and in this way the authors of Ref. [10] explained the small width of $X(3872)$, when interpreted as a $c \bar{c} q \bar{q}$ tetraquark.

\subsection{The spin part}

As the quarks and antiquarks are spin 1/2 particles, the total spin of a tetraquark can be $S=0,1$ or 2 . This can be obtained by first coupling two particles to each other and then couple the two subsystems together. Let us denote the intermediate coupling states between two quarks (antiquarks) by $S_{i j}$ for spin 0 (Scalar) and by $A_{i j}$ for spin 1 (Axial). For a quark-antiquark pair we denote the states by $P_{i j}$ for spin 0 (Pseudoscalar) and by $V_{i j}$ for spin 1 (Vector). We need their permutation symmetry properties under a given transposition $(i j)$. For quark (antiquark) pairs we have

$$
(12)\left|S_{12}\right\rangle=-\left|S_{12}\right\rangle, \quad(12)\left|A_{12}\right\rangle=+\left|A_{12}\right\rangle
$$

and

$$
(34)\left|S_{34}\right\rangle=-\left|S_{34}\right\rangle, \quad(34)\left|A_{34}\right\rangle=+\left|A_{34}\right\rangle
$$


For quark-antiquark pairs we have

$$
(13)\left|P_{13}\right\rangle=-\left|P_{13}\right\rangle, \quad(13)\left|V_{13}\right\rangle=+\left|V_{13}\right\rangle,
$$

and

$$
(24)\left|P_{24}\right\rangle=-\left|P_{24}\right\rangle, \quad(24)\left|V_{24}\right\rangle=+\left|V_{24}\right\rangle .
$$

For $S=0$ there are two independent basis states for each channel. The bases associated to (44), (5) and (6) are

$$
\begin{array}{ll}
\left|A_{12} A_{34}\right\rangle, & \left|S_{12} S_{34}\right\rangle, \\
\left|P_{13} P_{24}\right\rangle, & \left|\left(V_{13} V_{24}\right)_{0}\right\rangle, \\
\left|P_{14} P_{23}\right\rangle, & \left|\left(V_{14} V_{23}\right)_{0}\right\rangle,
\end{array}
$$

For $S=1$ there are three independent states in each channel, to be identified by three distinct Young tableaux. As an example we give the basis for the direct meson-meson channel [14]

$$
\left|\left(P_{13} V_{24}\right)_{1}\right\rangle, \quad\left|\left(V_{13} P_{24}\right)_{1}\right\rangle, \quad\left|\left(V_{13} V_{24}\right)_{1}\right\rangle .
$$

The lower index indicates the total spin 1.

The case $S=2$ is trivial. There is a single state

$$
\chi^{S}=\left|\left(V_{13} V_{24}\right)_{2}\right\rangle
$$

which is symmetric under any permutation of particles.

\section{CHARGE CONJUGATION}

We deal with the ground state, $i$.e. we have $J=S$. Making the identification $1=$ $c, 2=q, 3=\bar{c}$ and $4=\bar{q}$, introduced above, it is convenient to first couple 1 to 3 and 2 to 4 and then the subsystem 13 to 24, as in Fig. 1b. The charge conjugation is equivalent to applying the permutation $(13)(24)$ to the wave function.

\subsection{Tetraquarks}

The colour state (5) does not change under the permutation $(13)(24)$. The discussion holds for spin states only.

From the properties (9) and (10) one can see that the spin states (12) and (15) remain unchanged under the permutation $(13)(24)$. It follows that the states $J^{P}=0^{+}$and $J^{P}=2^{+}$have charge conjugation $C=+$.

For $J^{P}=1^{+}$the situation is slightly more complicated. There are six linearly independent basis vectors built as products of colour (5) and spin (14) states.

$$
\begin{array}{ll}
\alpha_{1}=\left|1_{13} 1_{24}\left(P_{13} V_{24}\right)_{1}\right\rangle, & \alpha_{2}=\left|1_{13} 1_{24}\left(V_{13} P_{24}\right)_{1}\right\rangle, \\
\alpha_{3}=\left|1_{13} 1_{24}\left(V_{13} V_{24}\right)_{1}\right\rangle, & \alpha_{4}=\left|8_{13} 8_{24}\left(P_{13} V_{24}\right)_{1}\right\rangle, \\
\alpha_{5}=\left|8_{13} 8_{24}\left(V_{13} P_{24}\right)_{1}\right\rangle, & \alpha_{6}=\left|8_{13} 8_{24}\left(V_{13} V_{24}\right)_{1}\right\rangle .
\end{array}
$$


Under the permutation (13)(24) the basis vectors $\left|\alpha_{1}\right\rangle,\left|\alpha_{2}\right\rangle,\left|\alpha_{4}\right\rangle,\left|\alpha_{5}\right\rangle$ change sign thus have charge conjugation $C=-$. On the other hand $\left|\alpha_{3}\right\rangle$ and $\left|\alpha_{6}\right\rangle$ do not change sign, thus have charge conjugation $C=+$. Also, by construction, they correspond to tetraquarks where the spin of the $c \bar{c}$ pair is $S_{c \bar{c}}=1$.

All states $\alpha_{i}$ of Eq. (16) used to construct Table 1 of Ref. [10] have inadvertently been associated to $C=+$, as explained in Ref. [18].

\subsection{Meson-meson molecules}

The only interesting case is $J^{P}=1^{+}$. Here we need the basis states in the

exchange channel corresponding to Fig. 1c. As for the direct channel there are six linearly independent basis vectors

$$
\begin{array}{ll}
\beta_{1}=\left|1_{14} 1_{23}\left(P_{14} V_{23}\right)_{1}\right\rangle, & \beta_{2}=\left|1_{14} 1_{23}\left(V_{14} P_{23}\right)_{1}\right\rangle, \\
\beta_{3}=\left|1_{14} 1_{23}\left(V_{14} V_{23}\right)_{1}\right\rangle, & \beta_{4}=\left|8_{14} 8_{23}\left(P_{14} V_{23}\right)_{1}\right\rangle, \\
\beta_{5}=\left|8_{14} 8_{23}\left(V_{14} P_{23}\right)_{1}\right\rangle, & \beta_{6}=\left|8_{14} 8_{23}\left(V_{14} V_{23}\right)_{1}\right\rangle .
\end{array}
$$

Using the Appendix C of Ref. [14] and the relations [15]

$$
\left|1_{14} 1_{23}\right\rangle=\frac{1}{3}\left|1_{13} 1_{24}\right\rangle+\frac{2 \sqrt{2}}{3}\left|8_{13} 1_{24}\right\rangle, \quad\left|8_{14} 8_{23}\right\rangle=\frac{2 \sqrt{2}}{3}\left|1_{13} 1_{24}\right\rangle-\frac{1}{3}\left|8_{13} 1_{24}\right\rangle,
$$

one can express $\beta_{i}$ in terms of $\alpha_{i}$ or vice-versa. In particular, one has

$$
\begin{gathered}
\beta_{1}=\frac{1}{6}\left(\alpha_{1}+\alpha_{2}\right)-\frac{1}{3 \sqrt{2}} \alpha_{3}+\frac{\sqrt{2}}{3}\left(\alpha_{4}+\alpha_{5}\right)-\frac{2}{3} \alpha_{6}, \\
\beta_{2}=\frac{1}{6}\left(\alpha_{1}+\alpha_{2}\right)+\frac{1}{3 \sqrt{2}} \alpha_{3}+\frac{\sqrt{2}}{3}\left(\alpha_{4}+\alpha_{5}\right)+\frac{2}{3} \alpha_{6}, \\
\beta_{3}=-\frac{1}{3 \sqrt{2}}\left(\alpha_{1}-\alpha_{2}\right)-\frac{2}{3}\left(\alpha_{4}-\alpha_{5}\right) \\
\beta_{4}=\frac{\sqrt{2}}{3}\left(\alpha_{1}+\alpha_{2}\right)-\frac{1}{6}\left(\alpha_{4}+\alpha_{5}\right)-\frac{2}{3} \alpha_{3}+\frac{1}{3 \sqrt{2}} \alpha_{6}, \\
\beta_{5}=\frac{\sqrt{2}}{3}\left(\alpha_{1}+\alpha_{2}\right)-\frac{1}{6}\left(\alpha_{4}+\alpha_{5}\right)+\frac{2}{3} \alpha_{3}-\frac{1}{3 \sqrt{2}} \alpha_{6}, \\
\beta_{6}=-\frac{2}{3}\left(\alpha_{1}-\alpha_{2}\right)+\frac{1}{3 \sqrt{2}}\left(\alpha_{4}-\alpha_{5}\right)
\end{gathered}
$$

From the properties of $\alpha_{i}$ one can infer that $\beta_{1}, \beta_{2}, \beta_{4}$ and $\beta_{5}$ do not have a definite charge conjugation. Contrary, $\beta_{3}$ and $\beta_{6}$ have $C=-$, because they are 
linear combinations of states with $C=-$. Inverting the above relations one can obtain $\alpha_{i}$ in terms of $\beta_{i}$. We have, for example

$$
\alpha_{3}=-\frac{\sqrt{2}}{6}\left(\beta_{1}-\beta_{2}\right)-\frac{2}{3}\left(\beta_{4}-\beta_{5}\right), \quad \alpha_{6}=\frac{2}{3}\left(\beta_{1}-\beta_{2}\right)+\frac{\sqrt{2}}{6}\left(\beta_{4}-\beta_{5}\right) .
$$

Section 3.1 implies that both $\beta_{1}-\beta_{2}$ and $\beta_{4}-\beta_{5}$ have $C=+$. From the identification $1=c, 2=q, 3=\bar{c}$ and $4=\bar{q}$ and the definitions (17) it follows that

$$
\beta_{1}=D^{0} \bar{D}^{* 0}, \quad \beta_{2}=D^{* 0} \bar{D}^{0}
$$

Thus one has

$$
\begin{aligned}
& \alpha_{3}=-\frac{\sqrt{2}}{6}\left(D^{0} \bar{D}^{* 0}-D^{* 0} \bar{D}^{0}\right)-\frac{2}{3}\left(\beta_{4}-\beta_{5}\right) \\
& \alpha_{6}=-\frac{2}{3}\left(D^{0} \bar{D}^{* 0}-D^{* 0} \bar{D}^{0}\right)+\frac{\sqrt{2}}{6}\left(\beta_{4}-\beta_{5}\right),
\end{aligned}
$$

where the second component is a hidden colour state with the same spin structure as $\left(D^{0} \bar{D}^{* 0}-D^{* 0} \bar{D}^{0}\right)$. It follows that a molecular structure with $C=+$ can be obtained only from the difference $\beta_{1}-\beta_{2}$ plus its hidden colour counterpart. Except for this part, our result is in agreement with the discussion given in Ref. [12], but in disagreement with the molecular interpretation of Ref. [11]. The hidden colour component with $C=+$ can influence the energy of the system at short separations [13]. Such contributions have been ignored in the simple molecular picture, because the molecules are point-like particles [11, 12, 17].

One can also find that

$$
\alpha_{1}+\alpha_{2}=\beta_{1}+\beta_{2}, \quad \alpha_{5}+\alpha_{6}=\beta_{4}+\beta_{5}
$$

which show that $\beta_{1}+\beta_{2}$ and $\beta_{4}+\beta_{5}$ have $C=-$. Thus the (open channel) molecular state with $C=-$ reads

$$
\beta_{1}+\beta_{2}=D^{0} \bar{D}^{* 0}+D^{* 0} \bar{D}^{0}
$$

again in agreement with Ref. [12]. Interestingly, its hidden channel counterpart, $\beta_{4}+\beta_{5}$ is completely decoupled from the open channel (30).

Acknowledgements I am grateful to Yoshi Fujiwara for pointiong out an anomaly in the relations presently numbered as Eq. (18).

\section{REFERENCES}

1. S. K. Choi et al. [Belle Collaboration], Phys. Rev. Lett. 91, 262001 (2003).

2. D. Acosta et al. [CDF II Collaboration], Phys. Rev. Lett. 93 (2004) 072001.

3. V. M. Abazov et al. [D0 Collaboration], Phys. Rev. Lett. 93 (2004) 162002.

4. B. Aubert et al. [BABAR Collaboration], Phys. Rev. D 71 (2005) 071103.

5. K. Abe et al. [Belle Collaboration], Phys. Rev. Lett. 94 (2005) 182002. 
6. B. Aubert et al. [BABAR Collaboration], Phys. Rev. Lett. 95, 142001 (2005).

7. K. Abe et al. [Belle Collaboration], Phys. Rev. Lett. 100, 142001 (2008).

8. E. S. Swanson, Phys. Rept. 429 (2006) 243.

9. S. Godfrey and S. L. Olsen, Ann. Rev. Nucl. Part. Sci. 58 (2008) 51

10. H. Hogaasen, J. M. Richard and P. Sorba, Phys. Rev. D 73 (2006) 054013.

11. M. B. Voloshin, Phys. Lett. B 579, 316 (2004).

12. Y. R. Liu, X. Liu, W. Z. Deng and S. L. Zhu, Eur. Phys. J. C 56 (2008) 63

13. D. M. Brink and F. Stancu, Phys. Rev. D 49 (1994) 4665.

14. D. M. Brink and F. Stancu, Phys. Rev. D 57 (1998) 6778.

15. F. Stancu, Oxford Stud. Nucl. Phys. 19 (1996) 1, Chapter 10.

16. L. Maiani, F. Piccinini, A. D. Polosa and V. Riquer, Phys. Rev. D 71 (2005) 014028.

17. M. B. Voloshin and L. B. Okun, JETP Lett. 23 (1976) 333; A. De Rujula, H. Georgi and S. L. Glashow, Phys. Rev. Lett. 38 (1977) 317; N. A. Törnqvist, Phys. Rev. Lett. 67 (1992) 556; Z. Phys. C61 (1994) 526; Phys. Lett. B590, (2004) 209 and references therein; T. E. O. Ericson and G. Karl, Phys. Lett. B309 (1993) 426.

18. F. Stancu, Proceedings of the 11th International Conference on Nuclear Reactions Mechanisms, Varenna, Italy, 12-16 June 2006, arXiv:hep-ph/0607077. 